\title{
F-ETX: a predictive link state estimator for mobile networks
}

\author{
Sébastien Bindel ${ }^{1}$, Serge Chaumette ${ }^{1, *}$, Benoît Hilt $^{2}$ \\ ${ }^{1}$ LaBRI, University of Bordeaux, France \\ ${ }^{2}$ MIPS, University of Haute Alsace, France
}

\section{Abstract}

Due to their inherent features, Mobile Ad Hoc Networks have proven their efficiency to exchange data between mobile nodes. The main issue in this type of a network is the delivery of data to a destination. Unfortunately, the mobility of nodes and the disturbances of the propagation channel lead to the increase of the loss rate, which undermines routing performances. To address this issue, routing protocols use link quality estimators as a metric. However, current estimators have been designed for static wireless sensor networks and are not suitable in case of mobility. In order to overcome this issue, a novel metric called Fast ETX is suggested, which gives a reliable and accurate link quality assessment. It is setup by four estimators which assess and predict the link state. In addition, we design a framework to integrate this multi-estimator metric into a routing protocol.

Received on 30 October 2015; accepted on 02 January 2016; published on 20 June 2016

Keywords: MANET, link quality, forecasting, routing

Copyright (C) 2016 Sébastien Bindel et al., licensed to EAI. This is an open access article distributed under the terms of the Creative Commons Attribution license (http://creativecommons.org/licenses/by/3.0/), which permits unlimited use, distribution and reproduction in any medium so long as the original work is properly cited.

doi:10.4108/eai.20-6-2016.151517

\section{Introduction}

Due to their inherent characteristics, including selforganization, scalability and their potential for managing load balancing, ad-hoc networks are the best choice to deploy on-the-fly a wireless network. They rely on the cooperation of stations to provide an affordable solution to share data or video streams.

Nodes are free to move with a potential high speed making the topology of Mobile Ad-hoc Networks (MANETs) dynamic. They also have to deal with specific constraints related to their environment and the desired application. This includes vehicular networks, so called VANETs, characterized by potential high speed nodes which could drive in an urban, suburban or freeway environment. Besides, unmanned systems are emerging and include aerial, terrestrial and maritime vehicles. For example, terrestrial vehicles also called Unmanned Ground Vehicle (UGV) represent an actual challenge since they accomplish missions in various environments.

Since distributed applications require cooperation of nodes, they are bounded by connectivity and reliability

${ }^{*}$ Corresponding authors. Email: serge.chaumette@labri.fr issues. They are partially solved by routing protocols ensuring an end-to-end communication with a multihop relaying technique. However, a relevant problem for routing protocols is the assessment technique of the link state in order to rank and select the best one. Indeed routing and applications performances in terms of delay and reception rate depend on the reliability of the link state assessment. The traditional hop count metric relates the cost of a path to the number of hops required to reach the destination. However, this approach is insufficient in wireless networks, as demonstrated by De Couto et al. [1].

To overcome the limitations of the hop count metric, a current approach relates the link state to the link quality. This approach takes into account the lossy and the dynamic of a link to determine its quality. Two kinds of Link Quality Estimator (LQE) have been proposed in the literature. The first one is based on a measurement performed at the physical layer during the packet reception. However, the correlation between the Packet Reception Ratio (PRR) and their assessments is not directly deductible at all, which limits their efficiency [2] [3] [4]. The second one includes estimators which perform measurements at upper layers and may assess the link according to application requirements. 
Related work has mainly designed estimators for Wireless Sensor Networks (WSNs), which limits their effectiveness in dynamic networks. For example, most of them use either a fixed historic or a data filter, which limits the reactivity of the estimators. There is a need for a reliable and reactive estimator for mobile networks.

In order to overcome the limitations of current LQEs in a highly mobile environment, we suggest a novel link quality estimator which performs at the MAC layer. Our approach is based on a dynamic history to deal with the unpredictable link lifetime. In opposition to other traditional estimators, ours adapts its assessment according to the estimated link lifetime. Indeed, we assess the link lifetime also referred to the link stability in order to adapt the link quality evaluation. In practice, the history of the packet reception is maintained through a window, whose length determines the volume of acquired data. The handling of a predefined static window size limits both the reactivity and the accuracy (the number of values) of the assessment. This issue can be solved with the use of a dynamic window size. Its length is re-sized according to the measured link stability in order to adjust the reactivity and the accuracy of the assessment.

We have suggested [5] a LQE to address the problem of the link quality assessment in mobile networks. It has been shown as more reactive and accurate than the Expected Transmission Count (ETX) metric [1]. From experiments, we observed that an estimation expressed by a single value provides only a short-term vision and remains insufficient when several links need to be compared. Figure 1 shows the fluctuation of link quality, while a short-term estimation (represented by the couple of points on the plot) leads to selecting continually different link, once its quality becomes worse than another. At the opposite, a long term estimation (represented by the couple of arrows on the plot) can highlight a tendency of the link quality meanwhile one grows (Link $N^{o} 1$ ) and another decreases (Link $N^{o} 2$ ). In the second related work [6] we improved our LQE with the development of additional estimators assessing multiple features of a link in order to provide a reliable vision of the link state. This paper is an extended version of this work which provides additional assessment on the estimator performances and describes a framework in order to integrate our final metric called F-ETX (LQE + its companion estimators) with routing protocols. We increase the level of description of our solution and submit an innovative integration process of F-ETX in a routing protocol and show its benefits compared to other solutions. We have simulated realistic scenarios carefully and shown novel results confirming the performance of our metric.

The main contributions of this paper are :

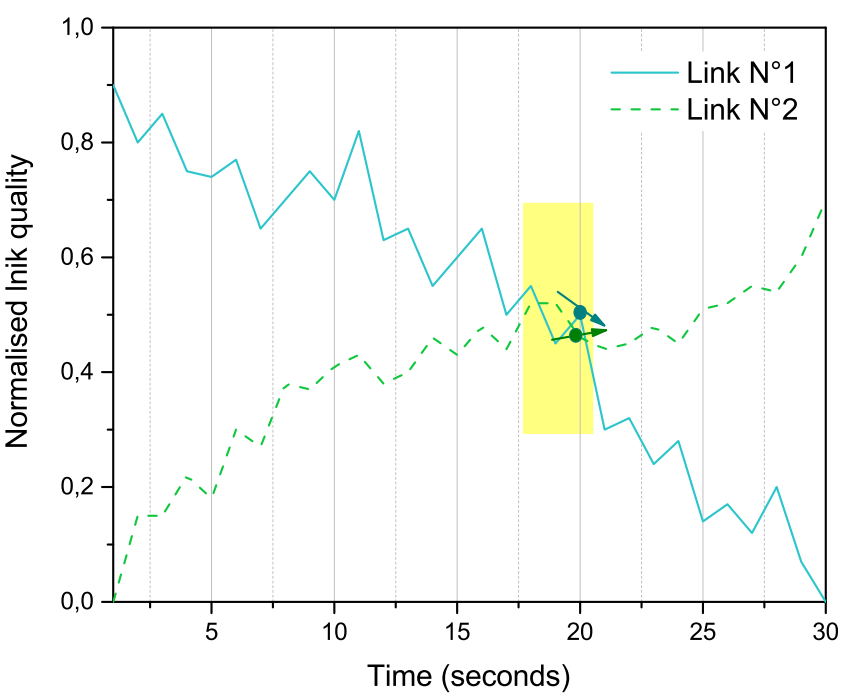

Figure 1. Linkselection: short vs long term

- A discussion concerning the challenges of LQEs in MANETs. We focus our attention on requirements needed for evaluating link quality estimators, especially to compare their performances. We survey the taxonomy and give a short analysis on estimators. Then, we give our challenges met during the development of our estimators (section 2).

- A detailed description of the design of our solution, including the link quality estimator, its companion estimators and also a framework to integrate the final metric into routing applications (section 3).

- A realistic evaluation of the efficiency of our solution within realistic simulations and provide detailed results to prove its usefulness (section 5).

The remainder of this paper is organised as follows. Section 2 presents the background including the link quality estimator requirements and an overview of current estimators. We detail in section 3 the design of our LQE, its companion estimators and we outline the framework to integrate the metric into a routing protocol. Section 4 describes realistic scenarios defined for our simulations. Their results are depicted in Section 5. At the end, we review the related work in Section 6.

\section{Background}

Evaluating the link quality in MANETs and its subsets is a challenging endeavour, especially with regards to the current practices, which develop most of link quality estimators for static wireless sensor networks. In this section, we detail some challenges to highlight 
problems of finding an efficient solution to assess the link quality and determine the link states in MANETs.

\subsection{Link Quality EstimatorsRequirements}

To get a reliable assessment of link quality, estimators have to meet requirements as described in [2].

Energy efficiency: energy is an essential concern, but is not required if nodes do not own a limited battery capacity, as in VANETs. It is nevertheless advisable for LQEs to minimise their computations and communication overhead. Complex estimation techniques and data structure such as learning might be inappropriate. About communication, even if an active link monitoring technique tends to consume much more power than passive link monitoring, a high beaconing rate should be avoided as it consumes too much energy.

Accuracy: the accuracy of a LQE is related to its ability to characterize the behaviour of a link. It has a significant impact on the protocol efficiency since the selection of the best link is relied on a comparison of available links.

Reactivity: it refers to the ability of an estimator to react quickly to a persistent change of the link quality. In wireless mobile networks, it is important for a LQE to be highly reactive in order to ensure an up-to-date estimation and also to detect quickly a link disruption. The reactivity depends on two factors : the monitoring window and the link monitoring scheme. The monitoring window is used to count received and lost packets in order to build an history of the packet reception. The monitoring scheme provides a tracking system based on three kinds of monitoring: active, passive and hybrid. In case of active monitoring, a node monitors a link by sending periodically a probe packet. In case of passive monitoring, a node exploits current traffic by overhearing the media without generating additional communication overhead. In a hybrid monitoring, a node uses both active and passive methods, thereby enabling an efficient balance between up-to-date measurement and overhead efficiency. Finally, the use of a reduced window and an active monitoring with an high beaconing rate leads to making a reactive $\mathrm{LQE}$.

Stability: unlike the reactivity property, it refers to the ability to tolerate transient variations of the link quality. Indeed, reactivity and stability are at odds. For instance, if a transient link quality change occurs, the routing protocol does not have to find another route, since routing computation involves an useless energy consumption. In [7], the authors argue that stability is met with a long term estimation. For example, EWMA filters with a large smoothing factor can deliver a long term estimation.

As a matter of fact, the design of a LQE meeting all quoted requirements is a challenging task. Indeed, reactivity and stability are at odds, meaning that a LQE cannot be both reactive and stable. Even if in MANETs LQEs have to be reactive, stability is also advocated to avoid for the protocol too often re-computation, e.g., routes change for routing protocols. The reliability of a LQE is relied on the accuracy, since the best link selection is based on a link quality comparison. Last but not the least, energy consumption deals with the lifetime of a node and must be considered, to have a trade-off between energy efficiency and estimation accuracy.

\subsection{Physical vs Logical information}

In order to assess the link quality estimation, an LQE retrieves information from either the physical layer or upper layers such as data link or network. Indeed, LQE can be classified in two categories: hardware-based and software-based.

Classical hardware-based estimators are based on the received signal properties such as the Received Signal Strength (RSSI), the Signal-to-Noise Ratio (SNR) and the Link Quality Indicator (LQI). Besides, these estimators read directly information from the radio transceiver ${ }^{1}$ to provide an estimation correlated with the Packet Reception Ratio (PRR) in order to observe the estimation accuracy. However, several research activities have shown their limitations to evaluate the link quality if it is situated in the transitional region. To overcome this drawback a novel kind of estimator retrieves information from the radio chip decoding process. The first one deals with the DSSS (Direct Sequence Spread Spectrum) decoding error analysis [8] [9] meanwhile a second one [10] extracts information from the OFDM (Orthogonal frequencydivision multiplexing) decoding process to assess the link state and predict future link disruptions. These estimators profit from information reported by the radio chip for making an accurate estimation of the link quality and even more the link state determination. Unfortunately, they impose by their computation to be set into specific radio chips, since they exploit data returned by the decoding process.

At the opposite, logical-based estimators assess the link quality according to the application point of view, i.e., the successful packet reception ratio or packet

\footnotetext{
${ }^{1}$ LQI is only available on specific radio chips for the IEEE 802.15 .4 wireless networks
} 
transmitted. Unlike hardware-based estimators, their assessments are directly correlated with the packet reception. They are able to evaluate the link quality not only through the PRR but also through the number of packets needed to ensure a successful transmission, called Required Number of Packet (RNP). Indeed, the estimation involves the computation of the up and downlink packet reception ratio by the sender which counts the number of packets transmitted and transmitted back. Another strategy adopted by the Expected Transmission Count (ETX) estimator is to maintain by the receiver-side the probability of the forward ratio and the backward delivery ratio via an active monitoring. Performance analysis shows that RNP/ETX based estimators are able to estimate the link quality with a better accuracy than PRR based, for the reason that RNP estimation takes into account the underlying distribution of packets loss. Thus, these estimators appear also more reactive by catching packet losses of the both sides of a link.

\subsection{Challenges}

The requirements detailed in section 2.1 are not sufficient to consider LQEs as suitable in mobile networks. In order to address the challenges of the link quality estimation in mobile networks, we detail the new challenges encountered.

- Support mobility and disturbances. The first challenge that we met is the support of the mobility of the nodes. Traditional estimators have been designed for static WSNs and do not support quick network topology changes. Thus, our estimators must be reactive enough to deal with the volatility of nodes. A mobile node may change its speed, location and environment. This leads to an important change in the signal disturbance via a different shadowing, fast-fading and Doppler effects. Our approach should take into account the volatility of a channel that encounters the nodes to ensure an up-to-date estimation but also the signal disturbance to deliver an accurate estimation of the link quality.

- Link selection. Most of LQEs assesses the quality through a single value that leads to catching only a snapshot. This expressiveness of a such estimation leads to comparing only short-term estimations without the possibility to take into account the dynamic of the link. There is a need to take into account this dynamic into the link quality assessment in order to ensure a reliable and robust link selection made by upper protocols.

- Integration. Protocols and estimators interact together through a single value to select the best link. Even if the multi-estimator approach assesses different features of the link with a multiple estimation, the results are fitted into a single one. This ensures a standard interaction but does not profit from a multi-estimation. Indeed, the protocol cannot adopt a strategy according to each estimation, that limits its efficiency. Our approach will ensure to take into account each estimation making the protocol more reactive to link state changes.

\section{Estimators Design}

In this section we describe our estimators and the framework designed to integrate them into a routing protocol. We detail in first the design of our LQE. Then, we present the companion estimators which complete the link quality estimation in order to provide a reliable link state assessment that is able to forecast the link behaviour. Current metrics which use a multi-estimation technique merge their different assessments into a single value. This would guarantee to be compatible with current routing protocols, because they use directly the results returned by estimators to adapt their routing policy. We argue this strategy is not the best way for the reason of multi-estimators assess different features of the link not directly related. To this end, we suggest a framework to integrate the link quality estimator and its companions with a routing protocol. The aim for the protocol is to profit from all estimators to trigger events as soon as they detect a link state change.

\subsection{Overview}

In recent years, a number of research efforts have been done to develop link quality estimators for WSNs. However, their performances are limited in a mobile environment. To overcome the identified issues described in section 2, we designed a novel link quality indicator based on a link quality estimation enhanced by additional estimators in order to predict the link state. Indeed, our estimators give both an instant assessment and a time based trend.

The proposed estimator is based on a dynamic window size used to build an history of the packets reception. Our approach makes our estimators suitable for dynamic and mobile environments. Even if our LQE is based on the ETX, it overcomes the main problem of the accuracy and the reactivity of the estimator. Indeed, ETX is based on a static window size, implying a tradeoff a between the reactivity and the granularity of the estimation. This problem is inherent to the use of a static window size as we shown in [5]. To solve this issue, the estimator uses a dynamic window size to adjust the link quality estimation to the link lifetime. Indeed, our estimator can estimate both the quality and 
the link lifetime also referred as the stability. We also developed additional estimators to make our estimator more robust and able to make predictions about the link state.

In order to fulfill our goals, we address the issue of the integration of our estimators with routing protocols. Current solutions such as F-LQE [11] and HoPS [12] merge the multi-estimations into a single metric. This approach tends to hide the property of each estimator. We propose to trigger specific routing mechanisms according to each assessment given by estimators.

\subsection{Windowmanagement}

Using a window allows to maintain an history of the packets reception state (received or lost) and its size sets the number of observed packets. Like ETX, our estimator is based on an active monitoring in which probe packets are periodically sent, that leads to filling or emptying the window at the same rate. Thus, the window size impacts both the reactivity and the accuracy of the assessment. The reactivity relies on the convergence time to declare a link disrupted or a link with a maximum quality (time to fulfill the window). Beside, the accuracy depends on the number of observed packet. Therefore, the use of a static window size implies a trade-off between accuracy and reactivity. We suggest the use of a window with a dynamic size in order to manage the history according to the link lifetime. Indeed, a node with an high velocity has a reduced link lifetime but can have an important link quality. Our main goal is to re-size the window as soon as a disruption occurs. To this end, two algorithms are required, one to reduce the window size and the other to grow the window.

Window Size Reduction Algorithm. As reactivity is a major concern for link quality metrics, F-ETX uses an algorithm which ensures both a fresh and an accurate estimation. Indeed, the algorithm maintains an history of packets, called window and tracks their reception state. Let a packet $p \in P$ a finite whole of observed packets such as, $P=\left\{p_{0}, p_{1}, p_{2}, \cdots, p_{n-1}\right\}$ with $n$ the number of observations. Each observed packet, $p$ is labelled according to its reception state such as, a label $L \in[0,1]$ in which, $L \leftarrow 0$ indicates a loss and $L \leftarrow$ 1 a reception. The number of packet considered as received, $a$, and lost $\bar{a}$ are computed as follows :

$$
\begin{aligned}
& n=a+\bar{a}, \\
& a=\sum_{i=1}^{n} L_{i}, \\
& \bar{a}=n-\sum_{i=1}^{n} L_{i} .
\end{aligned}
$$

Thus, $n$ is reduced according to the number of packet considered as lost :

$$
n=\frac{n}{2^{\bar{a}}}
$$

As shown in [5], in case of successive packet losses, the algorithm enables an exponential $\left(2^{\bar{a}}\right)$ decrease of $n$.

In praxis, maintaining a window let us create a history of packets identified with a unique ID such as a sequence number. A packet is declared received or lost according to a timer, also used to send periodically a probe packet. The figure 2 depicts an example of a window size reduction.

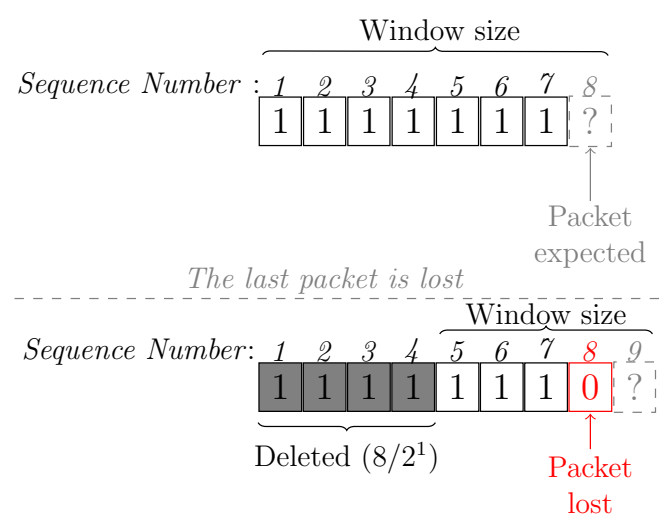

Figure 2. The windowsize reduction

The timer is scheduled to trigger a transmission of a probe packet every seconds. To avoid a synchronization among nodes, transmissions are delayed according to a random variable fixed at $[0,210] \mathrm{ms}$. We also consider the signal propagation delay as insignificant, for the reason that the celerity of an electromagnetic wave in the air is at $3 \times 10^{8} \mathrm{~m} \cdot \mathrm{s}^{-1}$. Therefore, a delayed reception cannot exceed one second.

Window Size Growing Algorithm. To ensure a suitable link quality estimation, it is required to adjust the estimation to the link stability. The suggested algorithm attempts to adjust the window by keeping or increasing its size. Indeed, once a packet is received, the algorithm determines if the window is increased or slid. To this end, a threshold $T_{h}$ is set as the window size, once a packet is declared as lost. Until the window size is lower than $T_{h}$, the widow size is incremented by one for each new packet received. Indeed, the algorithm tries to recover the last window size when a disruption occurs. This phase is called the recovery phase. If the window size is equals or higher than $T_{h}$, each new received packet increments a dedicated counter, $C$. Then, the window $(W)$ is increased or shifted (left) as described in the algorithm 1.

This approach limits the speed of the growth of the window size according to the loss occurrence and adjusts the accuracy of the estimation according to 


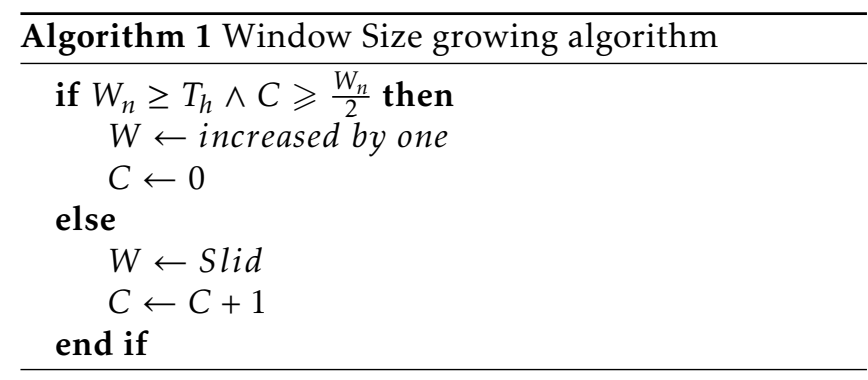

the link stability. In this phase, the algorithm senses the link stability and is called stability sensing. The figure 3 depicts the behaviour of the window with our algorithms.

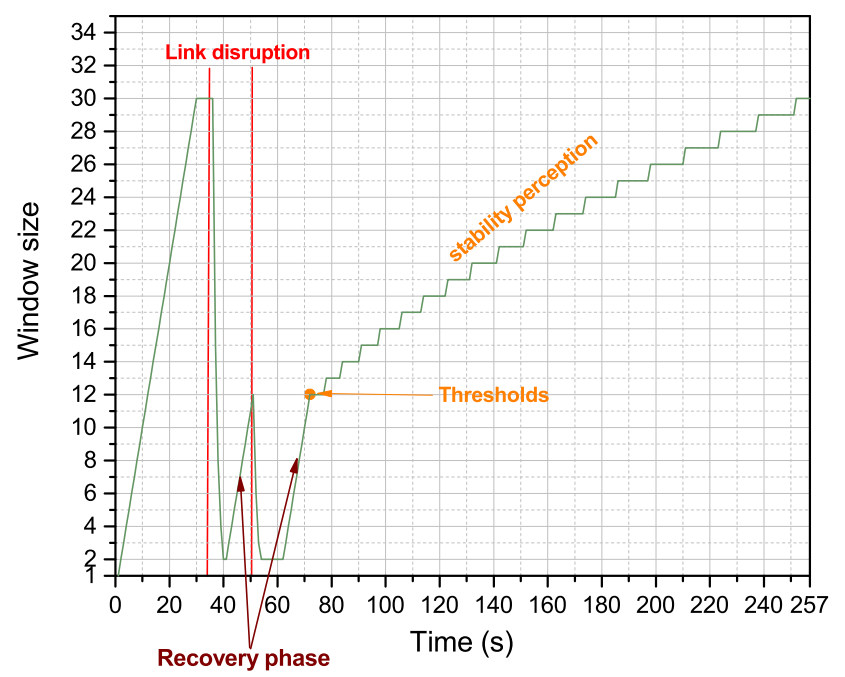

Figure 3. Behaviourof the windows

\subsection{Linkquality estimation}

The link quality assessment aims to find the highest throughput link. As for ETX, the estimation is based on a forward and a delivery ratio. The forward ratio $\left(d_{f}\right)$ measures the probability that a probe packet sent by a direct neighbour is received. The reverse delivery ratio $\left(d_{r}\right)$ measures the probability that a probe packet is successfully forwarded by a direct neighbour. Indeed, the previous sender hears its own probe packet and takes it as an acknowledgement. The data flow depicted in the figure 4 shows how the both ratios are computed.

The expected probability that a message is successfully received and acquitted is $d_{f} \times d_{r}$. If we consider a packet transmission as a Bernoulli trial (success or fail), the link quality estimation is determined by the probability to loss a packet. This probability is computed as follows:

$$
\chi^{L Q}=\frac{1}{\left(1-d_{f}\right) \times\left(1-d_{r}\right)} .
$$

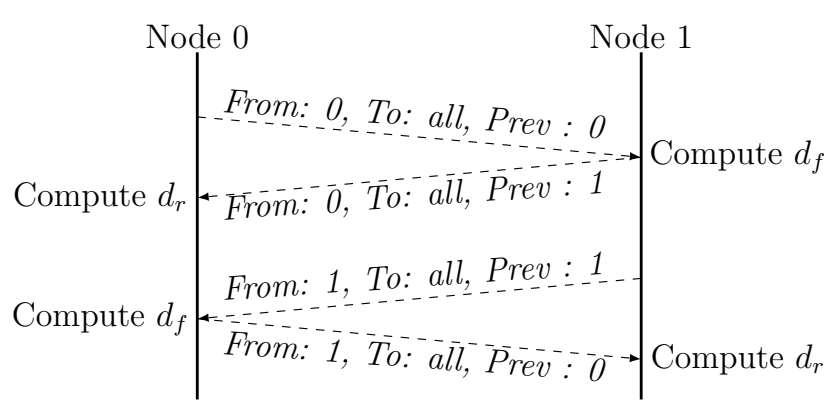

Figure 4. Case of probe transmsisions

We draw the attention to the fact that a couple of windows must be maintained, one dedicated to compute $d_{f}$ and another for the $d_{r}$ computation. Besides, an only timer is needed to schedule the sending of probe packets and update windows. To conclude, we give in the table 1 the convergence time of the classical ETX and our LQE to detect a link disruption, once the full windows.

Table 1. Detectiontime

\begin{tabular}{ccc}
\hline $\begin{array}{c}\text { Maximum } \\
\text { window's size }\end{array}$ & ETX & Our LQE \\
\hline 50 & $50 \mathrm{~s}$ & $5 \mathrm{~s}$ \\
30 & $30 \mathrm{~s}$ & $4 \mathrm{~s}$ \\
10 & $10 \mathrm{~s}$ & $3 \mathrm{~s}$ \\
\hline
\end{tabular}

\subsection{Companiorestimators}

Since a short term estimation is not sufficient, we developed three additional estimators, each evaluating a specific feature of the link to give a comprehensive assessment of the link state. These estimators relies on the Exponentially Weighted Moving Average (EWMA) to track the trend of each feature of the link.

Link quality trend. This indicator tracks the course of the link quality by computing the variation between the current $\chi_{t}^{L Q}$ and the previous estimation $\chi_{t-1}^{L Q}$. To provide a long term estimation the result is averaged with an EWMA.

$$
\begin{aligned}
& \Delta_{t}^{L Q}=\chi_{t}^{L Q}-\chi_{t-1}^{L Q}, \\
& \chi_{t}^{\text {Trend }}=\beta \times \Delta_{t}^{L Q}+(1-\beta) \times \chi_{t-1}^{\text {Trend }},
\end{aligned}
$$

the coefficient $\beta$ influences the sensitivity of the estimator. Choosing a small $\beta$ value is advisable to achieve a long term estimation. Note that two successive nulls $\chi^{L Q}$ indicate a disruption and reset the link quality trend estimator. 
Link stability estimation. We observed that a fine analysis of the windows content provides a link stability information. Let a binary state $[0,1]$ that represents the reception state of an excepted packet in a window. We note $W_{\max }$ the maximum window size, $W_{n}$ the current window size and the $W_{i}$ the $i^{\text {th }}$ element in the window. The windows maintained to compute the $d_{f}$ and $d_{r}$ probabilities are respectively noted $W^{d_{f}}$ and $W^{d_{r}}$. The link stability indicator is computed with an EWMA filter tacking into account the absolute $\Xi$ and the relative stability $\xi$ :

$$
\begin{array}{r}
\Xi=\frac{\sum_{i=1}^{W_{n}^{d_{f}}} W_{i}^{d_{f}}+\sum_{i=1}^{W_{n}^{d_{r}}} W_{i}^{d_{r}}}{2 W_{\max }}, \\
\xi=\frac{\sum_{i=1}^{W_{n}^{d_{f}}} W_{i}^{d_{f}}+\sum_{i=1}^{W_{n}^{d_{r}}} W_{i}^{d_{r}}}{W_{n}^{d_{f}}+W_{n}^{d_{r}}}, \\
\chi_{t}^{\text {Stab }}=\Xi_{t} \times \gamma+(1-\gamma) \times \xi_{t} .
\end{array}
$$

The absolute estimation $(\Xi)$ computed from the maximum window size (fixed value) represents the absolute level of stability of the link. The relative estimation $(\xi)$ computed from the current window size (dynamic value) represents the relative stability. This third estimation gives the level of the link stability according to the current window size. This information is useful, since, for a same absolute value, the relative link estimation gives an additional assessment taking into account losses which took place recently. Both absolute and relative information are suitable to assess the link stability. They must be taken into account in the same way. Hence, we advise a $\gamma$ value fixed at 0.5 .

Unidirectional link level. This last estimator deals with the detection of bidirectional links becoming unidirectional. Current approaches like F-LQE with the ASL estimator, track the difference between the uplink and downlink reception rates. Such a method becomes inefficient if the link has a short life time or experiment an high level of packet losses. In this case, windows are not sufficient trained to give a trustworthy estimation. Our method overcomes this limitation by measuring the variation of the up and downlink reception ratios. This makes it independent of the window size and does not require any training periods. Let $W$ be a window and $W_{n}^{t}$ its size at time $t$. The variation of the reception ratio provided by the window $W$ at time $t$ is noted $\Delta_{t}^{W i n}$. The indicator is given by:

$$
\begin{aligned}
& \Delta_{t}^{W i n}=\sum_{i=1}^{W_{n}^{t}} W_{i}-\sum_{i=1}^{W_{n}^{t-1}} W_{i}, \\
& \chi_{t}^{U L L}=\chi_{t-1}^{U L L} \times \lambda+(1-\lambda) \times \varphi\left(\Delta_{t}^{d_{f}}, \Delta_{t}^{d_{r}}\right), \\
& \text { with } \varphi(x, y)=\left\{\begin{array}{ll}
-1 & x<0 \wedge y>0 \\
1 & x>0 \wedge y<0 . \\
0 & \text { else }
\end{array} .\right.
\end{aligned}
$$

To give a tendency we advise a $\lambda$ value fixed at an high value. A link may become unidirectional (e.g. nodes with different transmit power level) if the assessment becomes negative.

\subsection{RoutingIntegation Framework}

In this section, we describe the framework designed to integrate all estimators in a routing protocol. Each estimator assesses a specific property of a link in order to provide a different vision of a link state. It is a key concept for addressing issues of routing protocols. Thus, the routing protocol must have holds all the cards to select the best link in order to maximize the packet delivery ratio and minimize the delay. Current metrics using multi-estimators such as F-LQE [11] and HoPS [12] compute a scored quality link estimation in order to provide an unique assessment. Even if Baccour et al. [13] have implemented F-LQE in the Collection Tree Protocol (CTP), there are no silver bullets to compute an ultimate single estimation including all values returned by estimators.

To solve this issue, we propose a framework that is able to integrate each estimation into the routing process. Indeed, each estimator would be related to the routing table in order to indicate specific link state if a event occurs. Based on an active monitoring, each estimation is computed after the reception of a probe packet. Then, they assign their assessment to the associated entry into the routing table. The proposed framework is illustrated in the figure 5 including the routing protocol and our metric (including all estimators).

We consider the routing table as the intermediary part to integrate the metric into a routing protocol. Indeed, the metric assesses both the link quality and indicates the link state and store this information into the routing table. Then, the routing algorithm selects the best link according its quality and its state. Thus, we need to define how the quality is assessed and which state is stored in the routing table. In addition, we detail how a routing algorithm may interpret information stored in the routing table.

Local Link Evaluation. Our metric includes four estimators including a short term, long term link quality, stability estimator and unidirectional link indicator. 


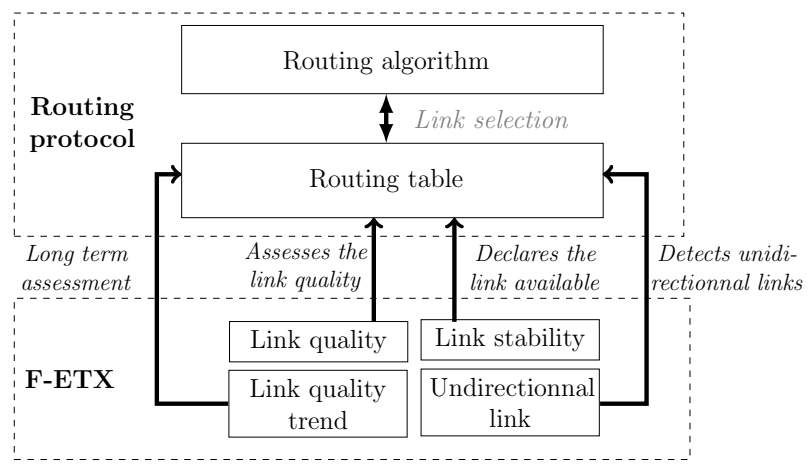

Figure 5. Routingframework

The unidirectional link indicator detects transient looses which turn a bidirectional link into an unidirectional link. Besides, persistent unidirectional links can be detected with a direct observation on the $d_{r}$ ratio indicating the number of packet retransmitted by a neighbour. Thus, our metric indicates two possible states indicating the unidirectional property of the link:

- $U_{p}$ : persistent unidirectional link

- $U_{t}$ : transient unidirectional link

The stability indicator provides information about the stability of the link. It enables to determine if a link can be relevant for routing data. This estimator is used to determine if a routing entry is valid or not. Indeed, a null estimation brands a routing entry as disable even if the quality is not null. Thus, our estimator indicates also two possible states on the link stability:

- $\chi^{\text {Stab }}=0$ : disable routing entry

- $\chi^{\text {Stab }}>0$ : available routing entry

As we shown in the figure 1, the major problem happens when the quality of two links are closed but have an opposite trend. Hence, we suggest an estimation merging both the short and the long term to assess the link quality, computed as follows :

$$
\text { Link quality }=\chi^{L Q}+\chi^{\text {Trend }}
$$

Taking into account both the short and the long term estimation, enables to penalize the short term estimation according to its current trend. If a couple of links has a close link quality, the metric is able to select the best one according to its quality trend.

Routing in Praxis. When a packet is received, the routing algorithm is responsible to route the packet to the destination by selecting the best link. This selection process is performed by selecting the corresponding entry in the routing table. That why, the protocol ranks for each destination node the potential best neighbour according to the link quality $\left(\chi^{L Q}+\chi^{\text {Trend }}\right)$. Then, the protocol inspects the stability indicator to determine if a route is declared available or disable. In the disable case, the algorithm selects the next route and restarts the same approach. At the end, the protocol checks if the link is unidirectional. In the case of a transient state, the route is selected, else the routing algorithm looks for another route.

\section{Simulation Settings}

To assess our approach, we use the ns-3 simulator [14]. We define two realistic scenarios including, a realistic mobility pattern (generated with BonnMotion [15]) and a signal propagation environment. The first scenario describes vehicular nodes moving in an urban area and communicate in an highly disturbed environment. In the second scenario, nodes evolve in a disaster area and go through predefined Point of Interests (POIs) and communicate in a free space environment.

\subsection{UrbanScenario}

In this scenario, 40 vehicles drive in an urban area of $200 m \times 200 m$ with a Manhattan mobility model $4 \times$ 4. We set the mean speed of vehicles at $30 \mathrm{~m} \cdot \mathrm{s}^{-1}$ in order to simulate an high speed traffic. From [16], we fix the channel propagation parameters, with a ThreeLogDistanceLossModel as a shadowing model and Rayleigh model as a fast-fading model, to recreate a realistic urban channel propagation environment. Table 2a details simulation parameters carefully.

\subsection{Disaster Scenario}

In this scenario, 40 vehicles are deployed on an freespace area of $275 \mathrm{~m} \times 250 \mathrm{~m}$ and move according to the Disaster mobility model, within nodes must inspect some Point of Interests (POI)s. From [16], we fix the channel propagation parameters, with a Friis loss model as a shadowing model and Nakagami model as a fast-fading model, to recreate a realistic free space propagation environment. Table $2 \mathrm{~b}$ details simulation parameters carefully.

\section{Simulation results}

We now describe the results given by our experiments and assess the performance of our approach. As mentioned in the description of our scenarios, we test our estimators in an urban and free space environment. We explore both the performance of (i) the quality estimator, (ii) the multi-estimators (iii) and the routing process using our framework.

\subsection{Performanceof the link quality estimator}

We proceed to investigate the performance of the ETX and the LQE of our F-ETX metric. We focus on three 
Table 2. Simulationparameters

(a) Urban Scenario

\begin{tabular}{lc}
\hline PHY parameters & \\
\hline Tx/Rx power $(\mathrm{dbm})$ & 0 \\
Gain of antenna $(\mathrm{dB})$ & 0 \\
Power Detection Threshold $(\mathrm{dbm})$ & -96 \\
\hline MAC parameters & \\
\hline Standard & $802.11 \mathrm{~g}$ \\
Mode & OFDM $6 \mathrm{Mpbs}$ \\
Rate adaptation & \\
\hline Propagation Loss Parameters & 2.5 \\
\hline ThreeLogDistance & 5 \\
Exponent 0 & 10 \\
Exponent 1 & 1 \\
Exponent 2 & 75 \\
Distance0 & 114 \\
Distance 1 & \\
Distance 2 & $\mathrm{m}=1$ \\
\hline Nakagami-m & \\
Rayleight & 5155236 \\
\hline Simulation Parameters & 9287851 \\
\hline Seed & \\
Run & \\
\hline
\end{tabular}

important aspects to asses the reliability of a estimator: the energy consumption, the stability and the reliability of the estimators.

Energy Consumption. In order to asses the energy consumption, we use an energy model retracing a Lithium Ion battery ${ }^{2}$ and a model retracing a typical energy consumption done by a wireless network interface ${ }^{3}$. The power consumptions of the model are summarized in the table 3 .

Table 3. Power consumptiomn Watts

\begin{tabular}{lc}
\hline Actions & Power consumption \\
\hline Transmission & 1.14 \\
Reception & 0.94 \\
Idle & 0.82 \\
Sleep & 0.10 \\
\hline
\end{tabular}

We are interested on how ETX and F-ETX stress the wireless interface, in term of transmissions required

\footnotetext{
2 see: ns-3 LiIonEnergySource model

${ }^{3}$ see: ns-3 WifiRadioEnergyModel
}

(b) Disaster Scenario

\begin{tabular}{lc}
\hline PHY parameters & \\
\hline Tx/Rx power $(\mathrm{dbm})$ & 0 \\
Gain of antenna $(\mathrm{dB})$ & 0 \\
Power Detection Threshold $(\mathrm{dbm})$ & -96 \\
\hline MAC parameters & \\
\hline Standard & $802.11 \mathrm{~g}$ \\
Mode & OFDM $6 \mathrm{Mpbs}$ \\
Rate adaptation & ARF \\
\hline Propagation Loss Parameters & \\
\hline Friss Propagation Model & $2.5 \times 10^{9}$ \\
Frequency $(\mathrm{Hz})$ & 1 \\
SystemLoss & 0 \\
MinLoss & \\
\hline Nakagami-m & 1.5 \\
m0 & 0.75 \\
m1 & 0 \\
m2 & 80 \\
d1 & 320 \\
d2 & 5155236 \\
\hline Simulation Parameters & 9287851 \\
\hline Seed & \\
Run & \\
\hline
\end{tabular}

to assess the link quality. We also observe the correlation between the number of nodes in the network and the energy consumed. The figure 6 shows the average amount of energy consumed by nodes, each measurement is marked with its standard-deviation. The graph shows that the energy consumption increases when the number of node expends. It results from the increase number of retransmission of probe packets required to assess the link quality. In the Disaster scenario, the energy consumed is more important than in the Manhattan scenario. Indeed, in the disaster Scenario the propagation environment disturbs less signals that increases the reception rate and by consequence the number of messages exchanged. A slight decrease on the plot of the Disaster scenario can be observed from 40 nodes in the network. It can be explained by the increase of the amount of transmitted data that leads to increasing the number of collisions and contribute to reduce the number of retransmitted packets. We conclude with ETX and our F-ETX have the same energy consumption profile, since they broadcast probe packets at the same frequency.

Stability. We proceed to investigate the stability of ETX and F-ETX that represents the ability of an estimator 


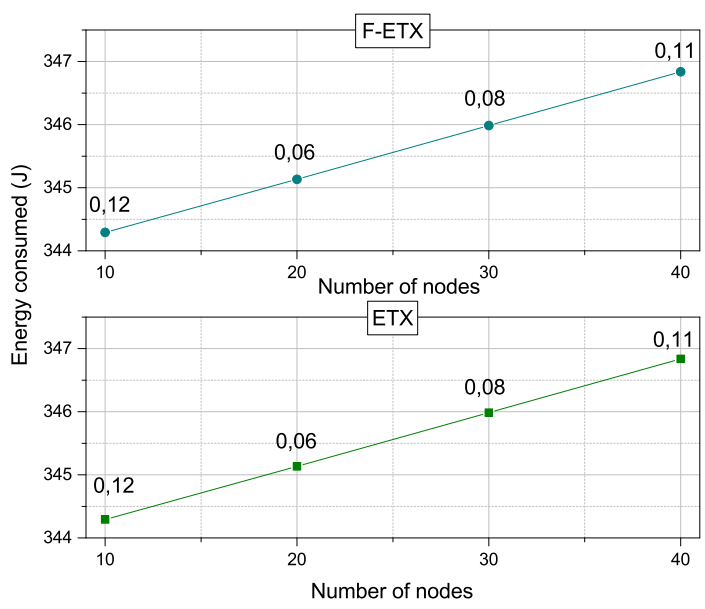

(a) Manhattan

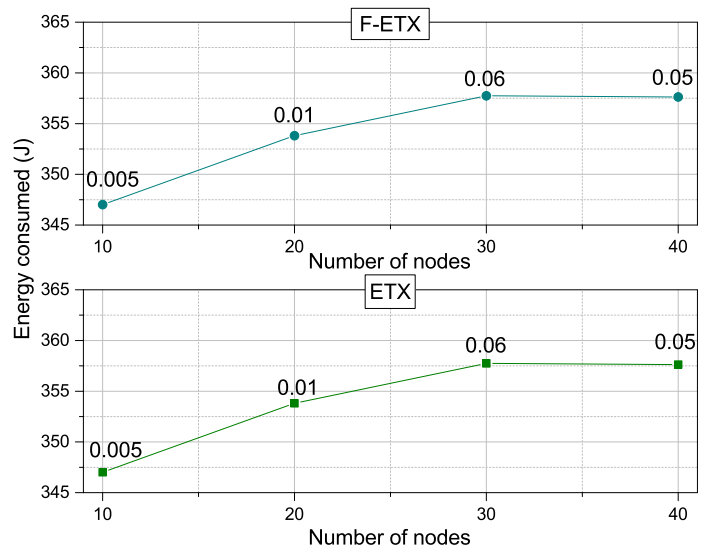

(b) Disaster

Figure 6. ETX vs F-ETX : Energy consumption

to resist to transient variations (short-term). We assess the stability by computing the coefficient of variation of the link quality estimation. It is defined as the ratio of the standard deviation to the mean. We computed this coefficient on each link presents in the network. In this experiment, we have defined a maximum window size of 15 Sequence Numbers. The figure 7 depicts the coefficient of variation of the couple of metrics.

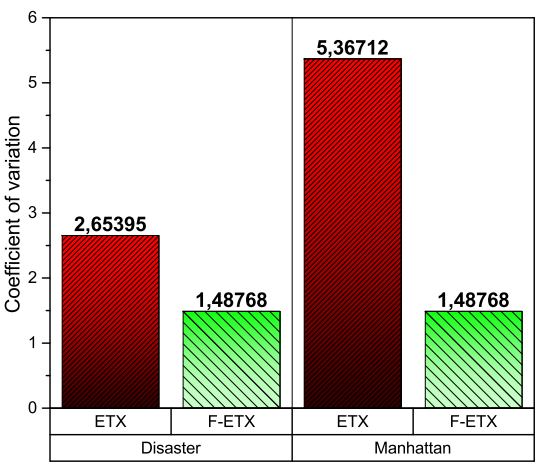

Figure 7. ETX vs F-ETX : Stability
ETX appears more unstable in Manhattan than in the Disaster scenario, since urban environment disturbs more the signal than in a free space (Disaster). However, F-ETX has the same stability in the both cases and is more stable than ETX.

Reliability. The reliability refers to the ability of the estimator to correctly assess the link quality. In order to evaluate this feature, we plot the empirical cumulative distribution function (CDF) and observe the distribution of estimations given by ETX and FETX. Our main goal is to determine if estimators overestimate and underestimate the link quality. The figure 8 depicts the empirical CDF. As the link quality is coded on 8 bits, the estimation is bounded between [0:255].

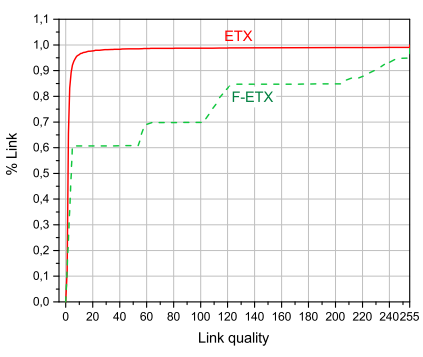

(a) Empirical CDF (Manhattan)

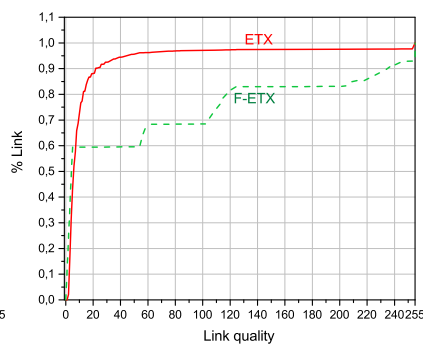

(b) Empirical CDF (Disaster)
Figure 8. ETX vs F-ETX: Reliability

We observe that F-ETX has a better distribution value than ETX in both case. ETX tends to underestimate the link quality with a large number of link estimated with a low quality. Beside, F-ETX declares less links with a low quality and tends not overestimate or underestimate the remainder of links. To this end, FETX appears as a more reliable estimator than ETX.

\subsection{Performanceof the multi-estimators}

We now investigate the performances and the robustness of all estimators of F-ETX. To this end, we compare the performance of our estimators and current metrics namely F-LQE [11] and HoPS [12]. We set the parameters of each estimator according to [11] for F-LQE and [12] for HoPS.

We fix the parameters of F-LQE as follows. We set the coefficient of the WMEWMA filter used to compute the packet delivery ratio at 0.6 . The assessment of the link quality and the link stability require an history of PRR that we set at 30. A minimal history is maintained at 5 PRR until to reach 30. About HoPS we set the parameters as follows. Coefficients are respectively set to 0.9 and 0.997 and its initializes short- and longterm estimations at $50 \%$ for new links. At the end, we determine for F-ETX the parameters of EWMA filters 


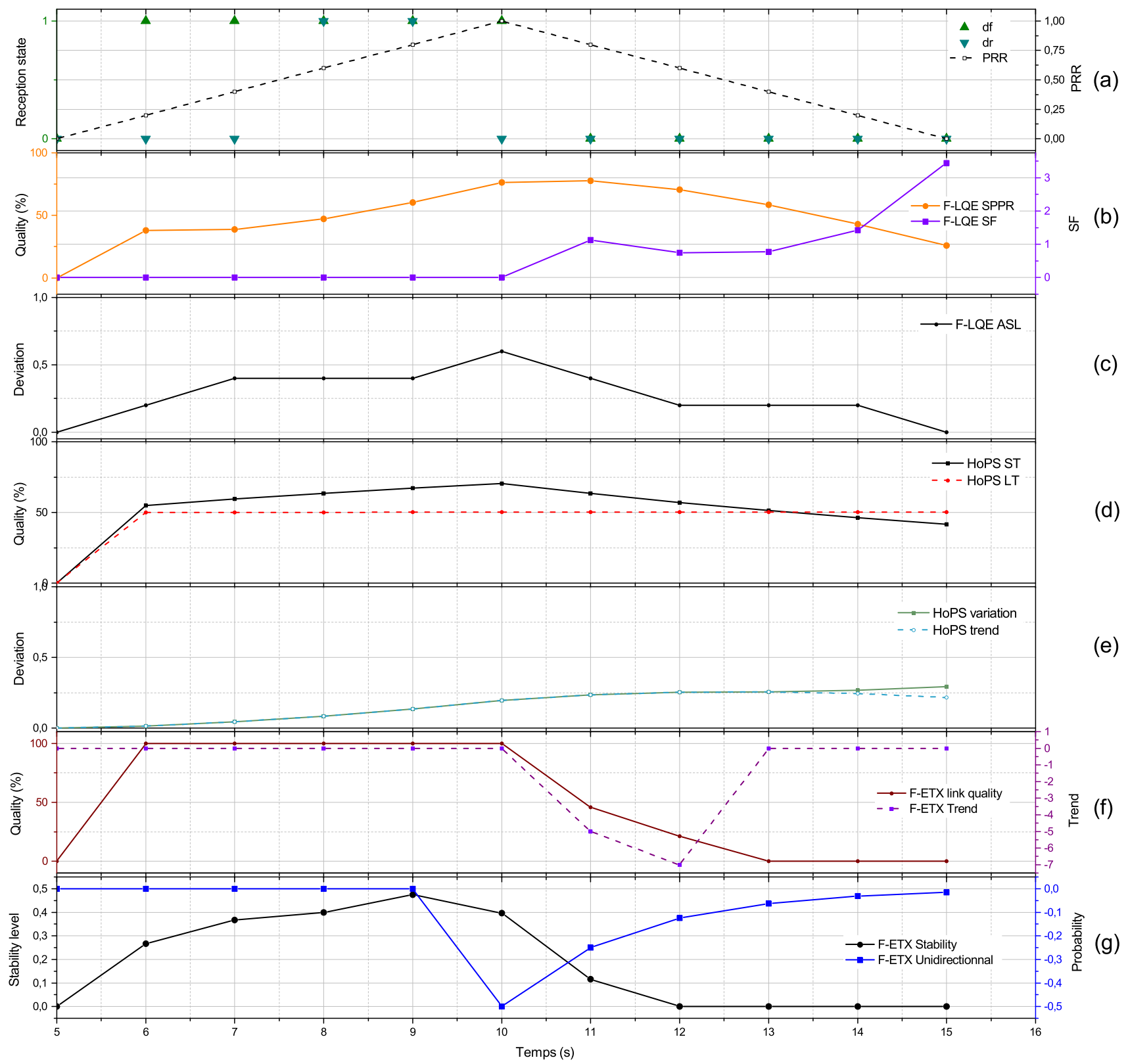

Figure 9. Fast speed crossing

used by companion estimators called $\lambda, \beta$ and $\gamma$ to 0.9 , 0.1 and 0.5 .

Our main goal is to assess both the agility of estimators by observing their ability to track fluctuation and their accuracy/robustness. We achieve both temporal and statistical evaluations. Through the temporal experiments, we observe the behaviour of estimators in order to have an overview on their ability to assess the link property. We made also a statistical evaluation of estimators to measure their forecasting properties.

Link Quality Tracking. The reliability of estimator were tested under two scenarios, involving two nodes. The first one takes place in an urban environment and defines a fast speed scenario wherein nodes are able to communicate during few time (4s). The second one takes place in a free space environment (Disaster) where nodes communicate longer during $19 \mathrm{~s}$.

Fast speed crossing: the figure 9 depicts the result of the first scenario. Regarding the distribution of the $d_{r}$ and $d_{f}$ in the figure 9(a) nodes are able to communicate during few time (from $6 s$ to $10 s$ while stochastic losses can be observed resulting from a significant fading (Rayleigh) effect). The PRR computed over an history of 5 packets declares the link disrupted at $15 \mathrm{~s}$, but the 


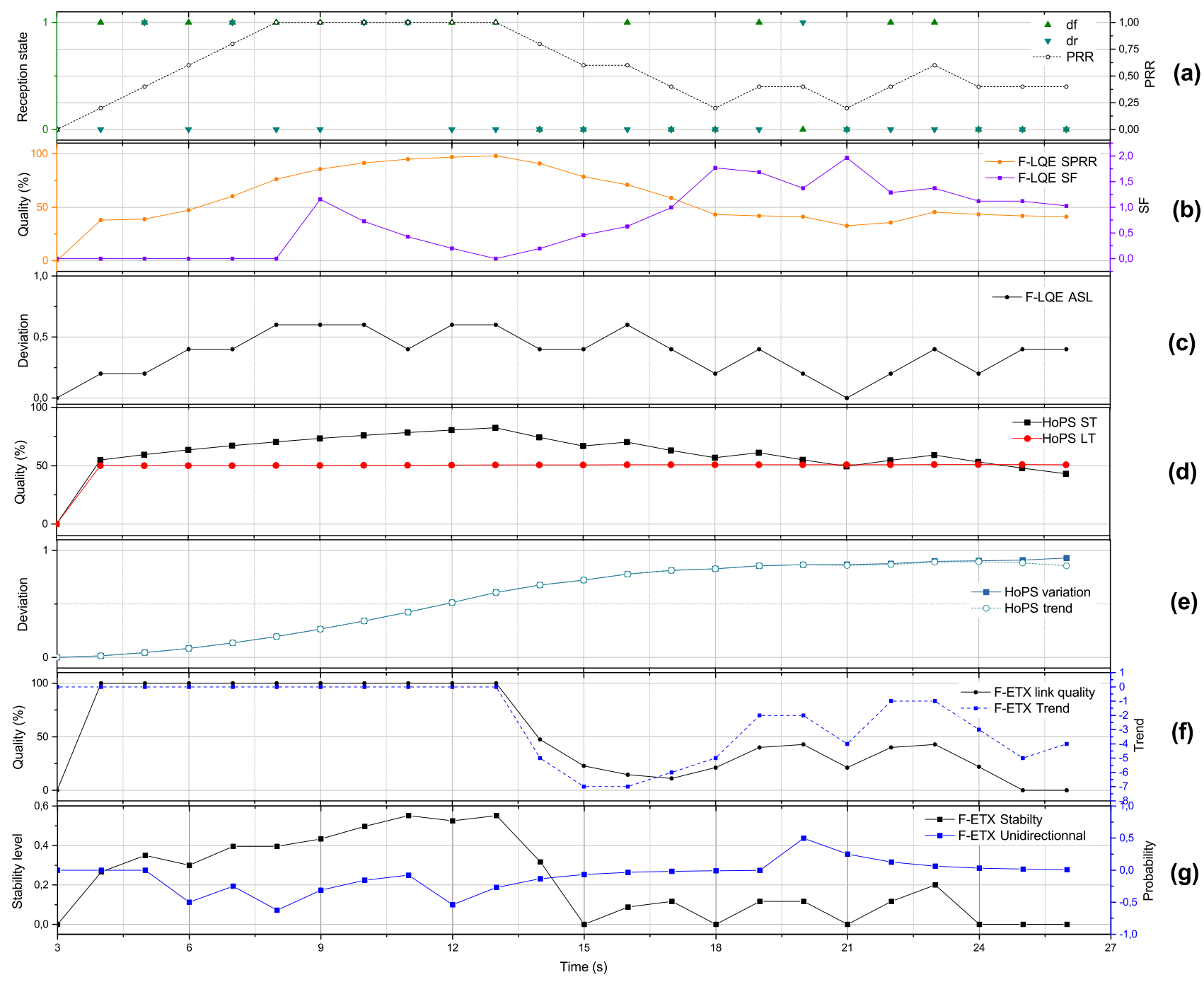

Figure 10. Low speed crossing

effective disruption occurs at $11 \mathrm{~s}$.

Concerning the F-LQE, figure 9(b) shows the smoothed PRR (SPRR) evaluating the link quality and the link stability estimation (SF). Below, the figure 9(c) shows the unidirectionality level of a link estimator (ASL). The SPRR follows the corresponding PRR (fig 9) trace with a smoothing trend, but the estimator is clearly not enough reactive and detect the disruption too late. This result from the EWMA filter that gives more stability than reactivity to the estimator. The SF estimator detects that the link quality is changing at $11 \mathrm{~s}$, because the link is disrupted. However, the variation indicated by the estimator does not reflect a disruption case but only a slight variation of the link quality. The disruption can be clearly detected at the end with a more important value of the indicator. About the ASL indicator, the variation of $d_{r}$ and $d_{f}$ distribution introduces light fluctuations indicating a low probability to have an asymmetric link.
Regarding estimators from HoPS (fig 9(d) and (e)) we observed the slow convergence time of the short-term estimation, while a link is disrupted, the estimator declares the link quality as not disrupted. But the longterm estimator indicates a correct decreasing trend. Consequently, the EWMA filter is well used for longterm estimation but is not suitable for a short-term estimation which smooth the estimation too. In the same manner, the link quality trend and the variation indicators are affected by the long reactivity of the short and long-term estimations and react too slowly when a disruption occurs.

The estimators of F-ETX are depicted in figure $9(\mathrm{f})$ and (g). In contrast with other LQEs, F-ETX is more reactive than the others and declares the link disrupted earlier (at 13s). The trend estimation indicates a degradation of the link quality via consecutive negative values. This is confirmed by the link stability estimator indicating a low level of stability and a decrease. We also 
observe that the stability estimator declares the link as disrupted earlier than the link quality estimator (12s). About the unidirectional indicator, its gives a positive value (at 10s) indicating that the link can be unidirectional.

Low speed crossing: the figure 10 depicts the result of the second scenario. The behaviours of $d_{r}$ and $d_{f}$ and the PRR indicate a progressive increase of the link quality until $13 s$ then the quality decreases progressively, fig $10(\mathrm{a})$.

Concerning F-LQE, the SPRR (fig 10(b)) increases progressively then decreases and confirms this tendency. The stability is correctly estimated by the SF estimator, indicating a decrease from 13s. However, the indicator indicates a false variation at $9 s$. About the ASL, stochastic losses disturb the estimator (fig 10(c)), that indicates the possibility of the link to be unidirectional.

About HoPs the short-term link quality estimator also indicates a correct tendency (fig $10(\mathrm{~d})$ ). We note that the HoPS ST increases and decreases faster than HoPS LT. But the HoPS trend and variation (fig 10(e)) take the same course at the beginning indicating an increase of the link stability then, the variation and the quality trend indicate a decrease of the link quality.

Regarding F-ETX (fig 10(f) and (g)) the link quality is estimated stable, since few packet are lost. This is confirmed by the trend estimator also indicating the same tendency then a decrease of the link quality. The same observation can be pointed out on the link stability indicator. The link unidirectionality level estimator of F-ETX indicates at $8 s$ and $12 s$ transients unidirectional property of link.

Statistical analysis. While previous evaluations give a detail about the strength and weakness of LQEs. We extend our assessment with a statistical analysis of all the links from both the Manhattan (fig. 11a) and the Disaster (fig. 11b) scenarios.

We have observed that the link quality estimator of FETX is more reactive than F-LQE and HoPs. In this statistical study we are focus on how this estimator can anticipate disruptions compared to the PRR solution based on an history of 5 packets. Figures 11a (a) and $11 \mathrm{~b}$ (a) show that F-ETX is clearly the best solution by anticipated disruptions before the PRR solution. Since it is based on the dynamic window size, the metric is more reactive and track link states changes very well. In addition, F-ETX assesses both link direction unlike a PRR solution that only evaluates the downlink.

The rest of the statistical analysis is made with the Mean Absolute Error (MAE) that measures the magnitude of the predicted estimation and the current outcome. A low score indicates a good prediction while a bigger value indicates a greater error between the prediction and the current value.
The link quality trend is an additional information that determines the current course of the link quality. Fig 11a (b) and 11b (b) show the link quality trend of HoPs and F-ETX. We observe the better ability of our estimator to give the tendency of the link quality compared to HoPS. Even if each of them is based on the link quality estimator and both are computed with an EWMA filter. Their abilities to track the link quality course depends on the ability of short-term link quality estimator. HoPS-ST suffers from lag with the use EWMA filter impacting the HoPS-LT. On the other hand, the link quality estimator from F-ETX is react but unstable. That is why with the use of EWMA the estimation is stabilized given a better longterm estimation than the HoPS-LT overestimating the tendency.

Figures $11 \mathrm{a}$ (c) and $11 \mathrm{~b}$ (c) show the unidirectional link estimator of the F-LQE and F-ETX. During the simulation, any effective unidirectional links are present. The ASL estimation makes a single reading on the reception ratio of the up and downlink often different when highly propagation disturbances are present. Our indicator adopts another strategy based on the variation between the up and downlink, as a result our estimation is more robust to disturbances and gives more suitable information about the potential of a bidirectional link to become unidirectional.

Tracking link stability is an essential feature to detect and differentiate transient and persistent links. We have compared in the figure $11 \mathrm{a}(\mathrm{d})$ and $11 \mathrm{~b}(\mathrm{~d})$ the variation of the value given by these estimators to the current variation observed from the delivery and forward ratios. F-ETX estimator gives the lowest MAE compared to the other. Because HoPS indicator only tracks the variation between the HoPs ST and LT estimations, that not really related with the link stability. About F-LQE, the estimation is based on a PRR history generating consecutive error predictions.

\subsection{Influenc on RoutingPerformances}

In the last experiment we are looking the influence of our metric on the routing performance. We enable both the AODV routing protocol and a developed protocol based on the BATMAN [17] routing protocol, using our framework. Five simultaneous communications run in the Manhattan and Disaster scenarios and we observe the packet delivery rate obtained with the AODV and with our protocol. The figure 12 depicts the obtained results and shows has the best performance of our solution in the both scenarios. Even if the propagation channel is highly disturbed in the Manhattan scenario, our approach is able to find a reliable path. Beside, in the disaster scenario, the free-space environment tends to disturb less the signal, explaining the better performance of protocols. 

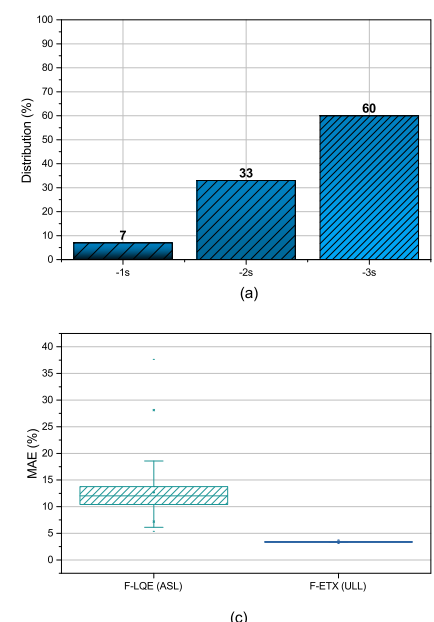

(a) Manhattan scenario
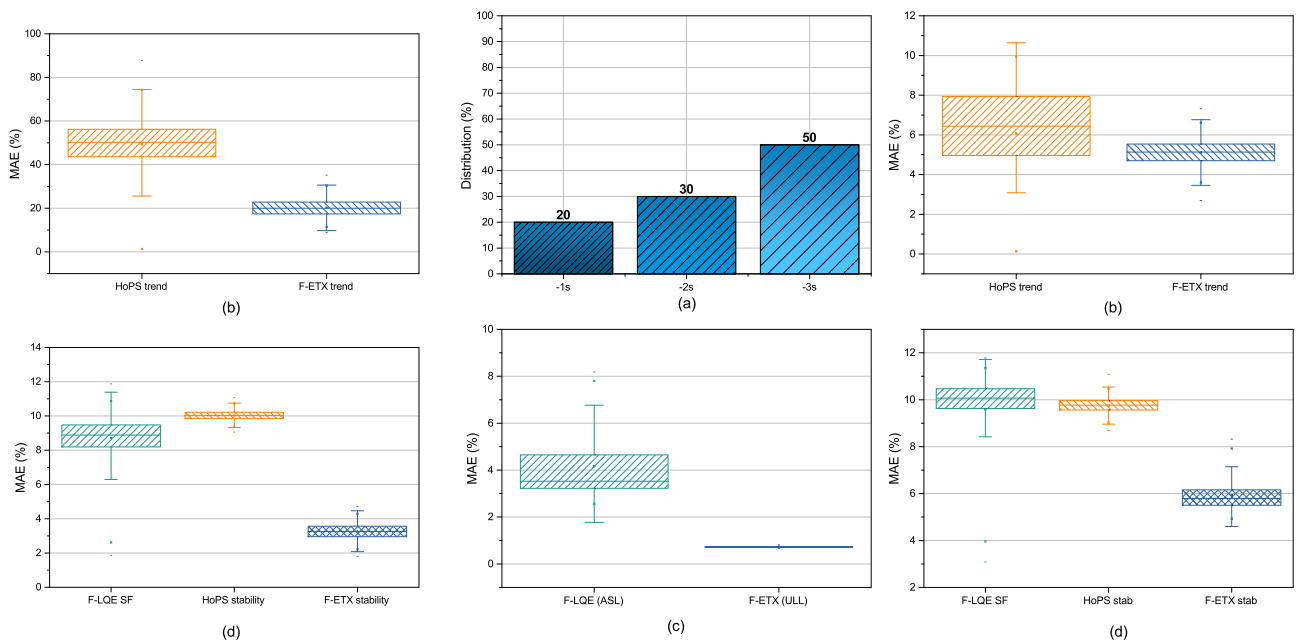

(d)
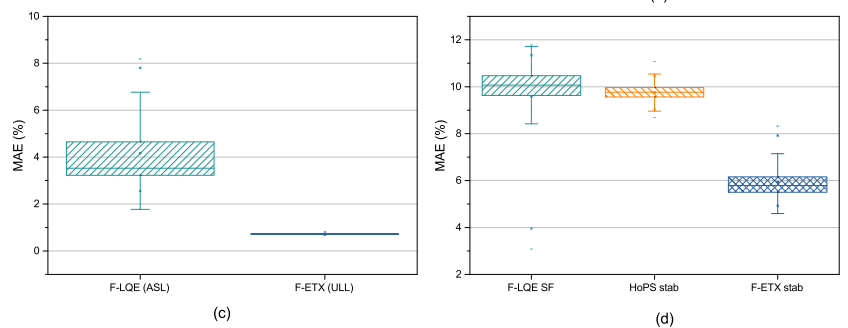

(b) Disaster scenario

Figure 11. Statistical evaluation

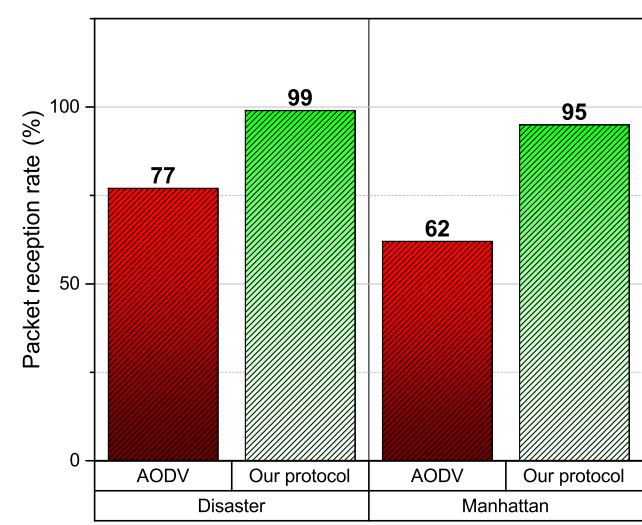

Figure 12. Packet delivery rate

\section{Related works}

Previous work suggested several logical LQEs. Although, proposed approaches are mainly designed for WSNs, our approach is still inspired by these estimators. In this section we describe these LQEs and highlight some observations from experiment.

Traditional metrics in wired networks, (i.e. hop count) fail to give an estimation on the paths reliability in wireless networks. De Couto et al. suggested the Expected Transmission Count (ETX) metric [18], which combines forward and backward packet reception ratios to compute a packet delivery success probability. Draves et al [19] experimented four type of metrics (ETX, hop count, Per-hop Round Trip Time and Perhop Packet Pair Delay) through a test bed placed in an indoor environment. They observed that ETX performs better than the others but in a mobility case, the performance of the hop count metric exceeds the ETX metric.
The Required Number of Packet (RNP) metric suggested by Cerpa et al [20] performs at the MAC layer and counts the average number of packet retransmissions required to deliver it successfully. They observed the temporal properties of low power wireless links and described the usefulness of such a metric to measure the link quality compared to a reception rate (RR). Unlike the RR information, RNP metric takes into account the underlying distribution of losses because the link quality is characterized in both directions. However, this metric requires an ARQ (Automatic Repeat-Request) mechanism to retransmit lost packets in order to determine the number of retransmission required to achieve a successful reception.

Woo et al [21] designed a routing protocol based on the channel snopping and introduced an agile filter called Window Mean with EWMA (WMEWMA) as an empirical estimator technique [22]. Authors showed its performance, over the others filters based on the Exponential Weighted Moving Average (EWMA).

An interesting novel approach supports that the link assessment can be made with multi-estimators to have a multi-faced vision of the link in order to compute a scored quality link estimation. Baccour et al. [11] designed an hybrid metric based on logical and physical information to provide multi-estimators that assess the packet delivery ratio, the link asymmetry level, the link stability and the channel quality. These estimators are aggregated into a single metric following a fuzzy logic method. In addition, they implemented F-LQE into the CTP routing protocol and proved its effectiveness in wireless sensor networks [13].

The Holistic Packet Statistic (HoPS) metric suggested by Renner et al. [12] incorporates four estimators; namely short-term, long-term, absolute deviation and trend estimation; computed with the EWMA filter. 
However an intrinsic problem of the use of this filter limits the agility of estimators. It also has the disadvantage to require a large amount of traffic to train the estimators and consequently increases the detection time of link state changes.

\section{Conclusion}

MANET networks are self-organizing mobile networks resulting from the cooperation of stations could be cars or unmanned vehicles (including aerial and terrestrial engines). The flexibility of these networks allow them to be deployed in several environments. However, these features tend to disturb the routing performance such as the packet delivery rate. To resolve this issue, protocols use link quality estimators but most of them were designed for static WSNs.

In order to deal with the inherent dynamic of MANETs, we proposed a link quality estimator based on a dynamic window size [5]. Then, we have developed additional estimators to reinforce the link quality estimation. Each of them evaluates a specific link feature: (i) the trend of the link quality, (ii) the link stability and (iii) the detection of unidirectional links. Indeed, these estimators complete the link quality assessment by evaluating different features of a link. The benefit of such approach is to provide a multifaced estimation on the link. Thus, the multi-estimator is able to determine and forecast the link state. Unlike current multi-estimators such as F-LQE [11] and HoPS [12] which mix each estimation into a single one, we propose a framework that enables the use of each estimator in the routing process. This approach makes the routing process efficient. Simulation results show that the link quality estimation given by F-ETX is more accurate and reliable than given by ETX. In addition, we have compared our multi-estimator with current approaches, by tracking their abilities to assess the link features. Besides, we highlighted prediction capabilities of our estimators through a statistical analysis that shows how our estimator overcomes other LQEs to predict link state changing. At the end of this paper, we showed the benefit our framework where the packet reception rate remains around $95 \%$ even if the signal is highly disturbed.

For future work, we plan to test the F-ETX and companion-metrics in other contexts such as building environments to confirm the suitability of our approach. In addition, tests in real environments will give us an important feedback on the estimator behaviour.

\section{References}

[1] De Couto, D.S.J., Aguayo, D., Chambers, B.A. and Morris, R. (2003) Performance of multihop wireless networks: Shortest path is not enough. SIGCOMM Comput. Commun. Rev. 33(1): 83-88.

[2] Baccour, N., KoubÂA, A., Mottola, L., ZúÑiga, M.A., Youssef, H., Boano, C.A. and Alves, M. (2012) Radio link quality estimation in wireless sensor networks: A survey. ACM Trans. Sen. Netw. 8(4): 34:1-34:33.

[3] Zhao, J. and Govindan, R. (2003) Understanding packet delivery performance in dense wireless sensor networks. In Proceedings of the 1st International Conference on Embedded Networked Sensor Systems, SenSys '03 (ACM): $1-13$.

[4] Srinivasan, K., Dutta, P., Tavakoli, A. and Levis, P. (2010) An empirical study of low-power wireless. ACM Trans. Sen. Netw. 6(2): 16:1-16:49.

[5] Bindel, S., Chaumette, S. and Hilt, B. (2015) F-etx: An enhancement of etx metric for wireless mobile networks. In Communication Technologies for Vehicles (Springer International Publishing), Lecture Notes in Computer Science 9066, 35-46.

[6] Bindel, S., Chaumette, S. and Hilt, B. (2015) A novel predictive link quality metric for mobile adhoc networks in urban contexts. In Ad Hoc Networks (Springer International Publishing), Lecture Notes of the Institute for Computer Sciences, Social Informatics and Telecommunications Engineering 155, 134-145.

[7] Lin, S., Zhou, G., Whitehouse, K., Wu, Y., Stankovic, J. and He, T. (2009) Towards stable network performance in wireless sensor networks. In Real-Time Systems Symposium, 2009, RTSS 2009. 30th IEEE: 227-237.

[8] Spuhler, M., Lenders, V. and Giustiniano, D. (2013) Blitz: Wireless link quality estimation in the dark. In Proceedings of the 10th European Conference on Wireless Sensor Networks, EWSN'13 (Springer-Verlag), 99-114.

[9] Heinzer, P., Lenders, V. and Legendre, F. (2012) Fast and accurate packet delivery estimation based on dsss chip errors. In INFOCOM, 2012 Proceedings IEEE: 29162920.

[10] Gabteni, H., Hilt, B., Drouhin, F., Ledy, J., Basset, M. and Lorenz, P. (2014) A novel predictive link state indicator for ad-hoc networks. In Global Communications Conference (GLOBECOM), 2014 IEEE: 149-154.

[11] Baccour, N., KoubÂa, A., Youssef, H., Ben JamâA, M., do Rosário, D., Alves, M. and Becker, L.B. (2010) Flqe: A fuzzy link quality estimator for wireless sensor networks. In Proceedings of the 7th European Conference on Wireless Sensor Networks, EWSN'10 (Springer-Verlag), 240-255.

[12] Renner, C., Ernst, S., Weyer, C. and Turau, V. (2011) Prediction accuracy of link-quality estimators. In $A C M$ EWSN.

[13] Baccour, N., KoubâA, A., Youssef, H. and Alves, M. (2015) Reliable link quality estimation in low-power wireless networks and its impact on tree-routing. Ad Hoc Networks 27: 1 - 25.

[14] Riley, G. and Henderson, T. (2010) The ns-3 network simulator. In Wehrle, K., GÃujneÅ§, M. and Gross, J. [eds.] Modeling and Tools for Network Simulation (Springer Berlin Heidelberg), 15-34.

[15] Aschenbruck, N., Ernst, R., Gerhards-Padilla, E. and Schwamborn, M. (2010) Bonnmotion: A mobility scenario generation and analysis tool. In Proceedings of the 
3rd International ICST Conference on Simulation Tools and Techniques, SIMUTools '10 (ICST (Institute for Computer Sciences, Social-Informatics and Telecommunications Engineering)): 51:1-51:10.

[16] Benin, J., Nowatkowski, M. and Owen, H. (2012) Vehicular network simulation propagation loss model parameter standardization in ns-3 and beyond. In Southeastcon, 2012 Proceedings of IEEE: 1-5.

[17] Collaborative authors, Batman open-mesh, http://www.open-mesh.org/projects/open-mesh/wiki.

[18] De Couto, D.S.J., Aguayo, D., Bicket, J. and Morris, R. (2003) A high-throughput path metric for multihop wireless routing. In Proceedings of the 9th Annual International Conference on Mobile Computing and Networking, MobiCom '03 (ACM): 134-146.

[19] Draves, R., Padhye, J. and Zill, B. (2004) Comparison of routing metrics for static multi-hop wireless networks.
SIGCOMM Comput. Commun. Rev. 34(4): 133-144.

[20] Cerpa, A., Wong, J.L., PotkonjaK, M. and Estrin, D. (2005) Temporal properties of low power wireless links: Modeling and implications on multi-hop routing. In Proceedings of the 6th ACM International Symposium on Mobile Ad Hoc Networking and Computing, MobiHoc '05 (ACM): 414-425.

[21] Woo, A., Tong, T. and Culler, D. (2003) Taming the underlying challenges of reliable multihop routing in sensor networks. In Proceedings of the 1st International Conference on Embedded Networked Sensor Systems, SenSys '03 (ACM): 14-27.

[22] Woo, A. and Culler, D. (2003) Evaluation of Efficient Link Reliability Estimators for Low-Power Wireless Networks. Tech. Rep. UCB/CSD-03-1270, EECS Department, University of California, Berkeley. 